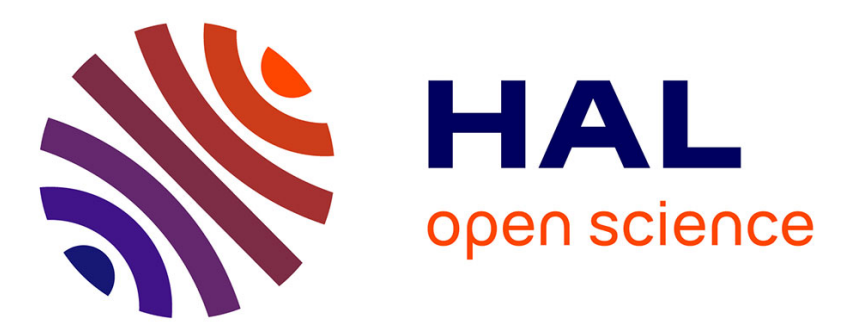

\title{
Towards a Driver Model to Clarify Cooperation Between Drivers and Haptic Guidance Systems
} Yishen Zhao, Philippe Chevrel, Fabien Claveau, Franck Mars

\section{To cite this version:}

Yishen Zhao, Philippe Chevrel, Fabien Claveau, Franck Mars. Towards a Driver Model to Clarify Cooperation Between Drivers and Haptic Guidance Systems. SMC 2020: IEEE International Conference on Systems, Man, and Cybernetics, Oct 2020, Toronto, Canada. 10.1109/SMC42975.2020.9283101. hal-02929620

\section{HAL Id: hal-02929620 \\ https://hal.science/hal-02929620}

Submitted on 3 Sep 2020

HAL is a multi-disciplinary open access archive for the deposit and dissemination of scientific research documents, whether they are published or not. The documents may come from teaching and research institutions in France or abroad, or from public or private research centers.
L'archive ouverte pluridisciplinaire HAL, est destinée au dépôt et à la diffusion de documents scientifiques de niveau recherche, publiés ou non, émanant des établissements d'enseignement et de recherche français ou étrangers, des laboratoires publics ou privés. 
Zhao, Y., Chevrel, P., Claveau, F., \& Mars, F. (in press). Towards a driver model to clarify cooperation between drivers and haptic guidance systems. Proceedings of the 2020 IEEE International Conference on Systems, Man, and Cybernetics.

\title{
Towards a Driver Model to Clarify Cooperation Between Drivers and Haptic Guidance Systems
}

\author{
Yishen Zhao ${ }^{1}$, Philippe Chevrel $^{1}$, Fabien Claveau ${ }^{1}$ and Franck Mars ${ }^{2}$
}

\begin{abstract}
Understanding a driver's behavior in a steering task is essential to the development of haptic guidance systems. This paper aims to predict driver torque control, especially when haptic guidance is part of haptic feedback. A new cybernetic driver model with an improved neuromuscular system is proposed and identified. It is assumed that the driver converts a target steering-wheel angle into torque by both indirect and direct control. Indirect control refers to the adaptation of the parameters of an internal model of steering compliance as perceived by the driver. Direct control accounts for the driver's corrective action through direct haptic feedback. The parameters of the model were identified with data collected from experiments conducted with a driving simulator. The results of identification were satisfactory and led to good representation of the driver's action, with or without haptic guidance. The model accurately predicted driver torque output. It can be used to study driver adaptation to haptic guidance systems.
\end{abstract}

Index Terms-cybernetic driver model, haptic feedback, neuromuscular system, parameter identification

\section{INTRODUCTION}

To meet the design guidelines for human-automation interaction, a specific mode has been proposed, called "shared haptic control." Shared haptic control has attracted increasing research attention in recent years [1], [2]. Its application to vehicle steering control is generally referred to as a "haptic guidance system." The main functionality of the system is to provide human drivers with continuous additional torque feedback through the steering wheel. Hence, the steering wheel is simultaneously controlled by the driver and the automated system so that it acts as an effective communication interface. The benefits of haptic guidance systems have been observed in lane-keeping performance [3]-[5].

To achieve sound cooperation between a driver and the haptic guidance system, it is essential to understand human behavior during a steering task. In several haptic guidance systems (e.g. see [6], [7]), a driver model is directly included to predict human driver behavior. A driver model can improve performance by minimizing the potential for conflict between the system and the driver. In addition, the model can analyze how drivers adapt their behavior when driving with the system.

A cybernetic driver model has been proposed in [8]-[10]. In this model, it is assumed that to steer the vehicle, the driver

\footnotetext{
${ }^{1}$ Yishen Zhao, Philippe Chevrel and Fabien Claveau are with Institut Mines-Télécom Atlantique, LS2N UMR CNRS 6004 (Laboratoire des Sciences du Numérique de Nantes), 44307 Nantes, France firstname.lastnamedls2n.fr

${ }^{2}$ Franck Mars is with CNRS \& Centrale Nantes, LS2N UMR CNRS 6004 (Laboratoire des Sciences du Numérique de Nantes), 44321 Nantes, France franck.marsels2n.fr
}

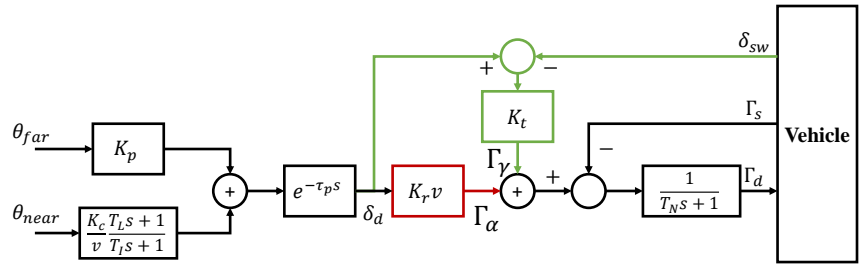

Fig. 1. Cybernetic driver model in [8]-[10].

implicitly aims at a steering angle $\delta_{d}$, which is determined from the visual scene (left part of Fig. 1). This angle is then converted to a torque applied to the steering system through co-activation of the muscles by $\alpha$ - and $\gamma$-motor commands. The model has been successfully used in the synthesizing a haptic steering system [11] and in discriminating various types of distraction through analyzing parametric variations [10], [12], [13].

However, the model has been designed and identified in situations where the human drives alone. It considers only the self-aligning torque as haptic feedback but does not take into account the influence of a haptic guidance system, which could be either parametric or structural. Generally, it is difficult for a driver to distinguish between self-aligning torque and haptic guidance torque because they act together on the steering wheel and are perceived through the same channel. Therefore, there is no reason to explicitly separate them in the driver model, and it is the combination that is called haptic feedback.

In addition, when analyzing the driver's neuromuscular behavior using the model, it becomes difficult to interpret the intermediate torque generated by the $\alpha$ - and $\gamma$-commands. Precisely, on the one hand, the co-activation of muscles must generate a torque that is

$$
\Gamma_{\alpha}+\Gamma \gamma \approx \Gamma_{s}+\Gamma_{d}
$$

as the time constant $T_{N}$ is relatively small. On the other hand, the total torque applied on the steering system, ignoring the friction, is

$$
\sum \Gamma \approx \Gamma_{d}-\Gamma_{s}
$$

Substituting the $\Gamma_{d}$ in (1) by the $\Gamma_{d}$ in (2) gives

$$
\Gamma_{\alpha}+\Gamma \gamma \approx 2 \Gamma_{s}+\sum \Gamma
$$

The above calculations imply that from the target steeringwheel angle $\delta_{d}$, the intermediate torque generated by the $\alpha$ and $\gamma$-commands is double the strength of the self-aligning torque. Such result has not been observed in any experiment yet. 


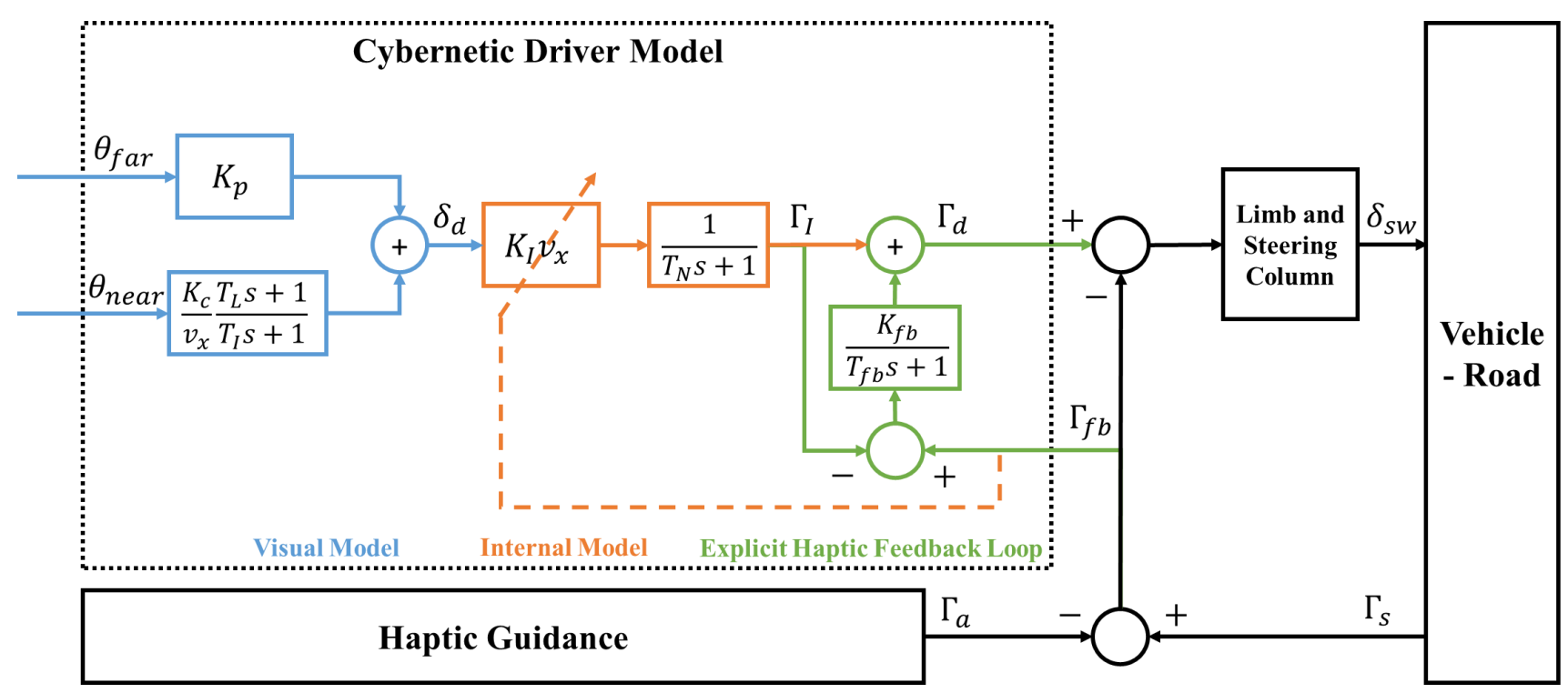

Fig. 2. Structure of the proposed cybernetic driver model. Blue: two-point visual model; orange: driver internal model; green: direct haptic feedback loop.

To solve these issues, this article presents a new driver model with a reconsidered neuromuscular system (NMS). The system is designed taking into account the haptic feedback, which is the sum of self-aligning torque and assistance torque when driving with the haptic guidance system, or only the former if driving alone. The structure of the model is discussed in Section II. The methods adopted for data collection and identification of the model parameters are presented in Section III and IV, respectively. The results are presented in Section V. Finally, we provide conclusions about the results and propose future work in Section VI.

\section{DRIVER Model With HAPTIC FEEDBACK}

The main contribution of this paper consists of the new driver model structure shown in Fig. 2. The description of signals is listed in Table I. This model decomposes the vehicle lateral-control task of driver into two steps: 1) generating a target steering-wheel angle by a two-point visual model; and 2) applying the target angle to limb-steering system by $N M S$, with the implicit and explicit involvement of haptic feedback, as shown by the dashed line and solid line from $\Gamma_{f b}$, respectively.

\section{A. Two-point Visual Model}

The two-point visual model is adopted as a starting point. As described in the literature [14], [15], the visual information that a human perceives while driving is extracted from both a distant and a near area. Two points within these zones, referred to as far and near points respectively, are a simplified representation of the visual inputs. The far point may be the tangent point of the inner edge of the road; it represents the anticipation of changes in the curvature of the road. The near point is a few meters ahead of the vehicle. It represents the visual perception of the lateral deviation of the vehicle used by the driver to keep the vehicle in the lane. The angle
TABLE I

DESCRIPTION OF SignALS IN Fig. 2

\begin{tabular}{c|c}
\hline \hline Signal & Description \\
\hline$\theta_{\text {far }}$ & Far-point Angle \\
$\theta_{\text {near }}$ & Near-point Angle \\
$\delta_{d}$ & Target Steering-wheel Angle \\
$\delta_{S W}$ & Actual Steering-wheel Angle \\
$\Gamma_{I}$ & Torque from Internal Model \\
$\Gamma_{d}$ & Driver Steering Torque \\
$\Gamma_{f b}$ & Haptic Feedback Torque \\
$\Gamma_{a}$ & Haptic Guidance Torque \\
$\Gamma_{s}$ & Self-aligning Torque \\
\hline \hline
\end{tabular}

between the heading of the vehicle and these points, namely $\theta_{\text {far }}$ and $\theta_{\text {near }}$, are used as input for the visual model.

\section{B. Neuromuscular Action}

Neuromuscular-system modelling aims to represent two fundamental mechanisms: 1) how muscles convert the target steering-wheel angle into steering torque and 2) how haptic feedback is involved in the driver's control [8]. It has been shown that humans can actually adapt to various types of haptic feedback laws but cannot drive without such feedback (i.e., with zero or inverted haptic feedback) [16]. In the proposed model, the NMS associated with the limb-steering system functionally acts as an angular servo system to ensure that the actual steering-wheel angle follows the target angle. The NMS may achieve this by direct or indirect control. "Indirect control" here refers to parameter adaptation. Specifically, the parameter defines an internal model of the steering system, simply reduced to its stiffness. By contrast, "direct control" consists of torque feedback that compensates for errors of the internal model. 
1) Driver Internal Model: There is reason to believe that drivers adapt their behavior through their interactions with the steering wheel, by perceiving steering compliance and inversing it. This idea has been proposed and explored in neural science [17]. The internal model results from driver perception and prior knowledge of the steering system. During driving, the arm muscles and the steering wheel are combined to form a limb-steering system. The driver learns about the dynamics of the system and adapts to them by updating an internal model of steering system compliance. Ideally, if the estimate is accurate (i.e. if the internal model is equal to the inverse steering system), no additional information is needed for the driver to reach the target steering angle; the driver can accurately provide the necessary steering torque. However, it is likely that this estimate is only approximate and corresponds to a simple (low-order) internal model [18]. In this study, it is limited to a static gain $K_{I} v_{x}$, which depends on the longitudinal speed $v_{x}$ of the vehicle.

An important characteristic of the internal model is that it can be learned by the driver through experience with the vehicle. The learning process can be rapid because an experienced driver can easily adapt to another vehicle with a different steering system. The same is true when the extra guidance torque is applied to the steering system. From a driver's perspective, it appears that the dynamics of the steering system is modified, and the driver thus adapts to cooperate with the new steering dynamics. To represent this process in the model, the haptic feedback signal is used to update the internal model. The adaptation of the internal model to the haptic guidance system should occur at the start of the driving scenario. Analysis of the speed of adaptation is beyond the scope of this study; only the adjustment mechanism of the internal model is considered.

The torque from the internal model is passed through a first-order system with a time constant $T_{N}$ to represent that muscles require time to produce the torque control $\Gamma_{I}$. The value of $T_{N}$ is fixed at $0.23 \mathrm{~s}$, which corresponds to the cut-off frequency of $0.7 \mathrm{~Hz}$ for reflexive muscle activity previously proposed [19].

2) Explicit Haptic Feedback Loop: As stated above, the internal model that the driver learned can hardly be equivalent to the inverse limb-steering dynamics. To compensate for the error between the internal model's output $\Gamma_{I}$ and the driver steering torque $\Gamma_{d}$, a closed-loop control must be formed. This control uses signals perceived by the driver from the steering wheel. From the perspective of muscle anatomy and physiology, muscles contain two types of sensors that convert mechanical stimuli into neural activity. The first is the muscle spindle, which detects changes and the change rate of muscle length. The second is the Golgi tendon organ, which detects changes in muscle force. In other words, either the steering-wheel angle $\delta_{S W}$, steering-wheel angular speed $\dot{\delta}_{S W}$ or the haptic feedback $\Gamma_{f b}$ can be used to establish the closed-loop control.

In our model, an explicit haptic feedback loop is introduced based on the difference between the haptic feedback
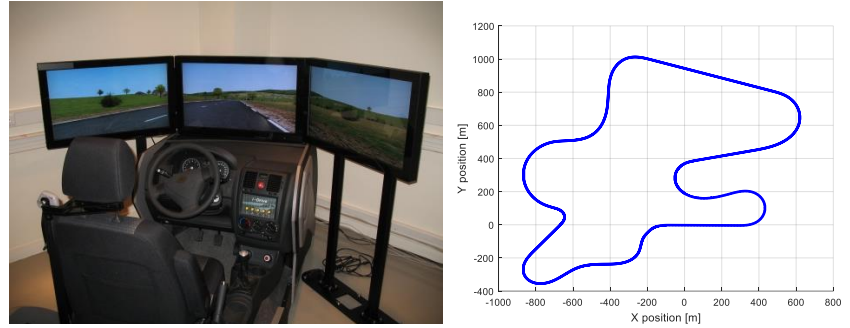

Fig. 3. Left: fixed-base driving simulator; right: track used in the experiment.

torque and the output torque of the internal model (i.e., $\left.\Gamma_{f b}-\Gamma_{I}\right)$. Other choices could have been $\delta_{d}-\delta_{S W}$ or $\dot{\delta}_{d}-\dot{\delta}_{S W}$. However, haptic feedback compensation is considered to be faster and more intuitive for the driver, while a minor error between the target steering angle and the actual steering angle would be more difficult to detect through muscle sensors and would likely be compensated for by visual information. For example, a small static error in the steering-wheel angle will gradually lead to a lane change and will eventually be corrected by the driver. In other words, the $N M S$ is more likely to counterbalance the haptic feedback to stabilize the steering wheel than to achieve the exact target steering-wheel angle.

\section{DATA ACQUiSITION}

\section{A. Apparatus}

An experiment for acquiring data was performed using a fixed-base driving simulator powered by SCANeR Studio (Fig. 3, left). It simulates the interior environment of a family car, with a complete dashboard; a five-speed gear stick; gas, brake and clutch pedals; and a steering wheel connected to a TRW steering system. Sensors for measuring steering-wheel angle, speed and torque are mounted in the steering system. The visual scene is displayed on three LCD screens: a central one in front of the driver and two others oriented at $45^{\circ}$ relative to the center. The screens cover a field of view of $25^{\circ}$ high and $115^{\circ}$ wide. In the experiment, a small family car, the Citroën C5, was chosen as the vehicle model.

\section{B. Haptic Guidance System}

A haptic guidance system was implemented in the driving simulator using a controller designed in Simulink. The controller was previously developed [7] and its global architecture is shown in Fig. 4. The final guidance $\Gamma_{a}$ was a combination of anticipatory and compensatory assistance. The anticipatory assistance generates a reference trajectory (references for states, $x_{r e f}$, and control input, $u_{r e f}$, of vehicleroad model) from previewed road curvature, $\rho_{\text {previewed }}$,

$$
\left(\begin{array}{l}
\Gamma_{\text {ref }} \\
x_{\text {ref }}
\end{array}\right)=K_{F F}(p) \rho_{\text {previewed }}
$$

where $K_{F F}(p)$ represents the transfer function of the trajectory generator. The applied anticipatory torque was then determined by accounting for the targeted sharing level: $\Gamma_{a_{\text {ref }}}=\alpha_{a n t} \Gamma_{r e f}$. The compensatory assistance adjusted 


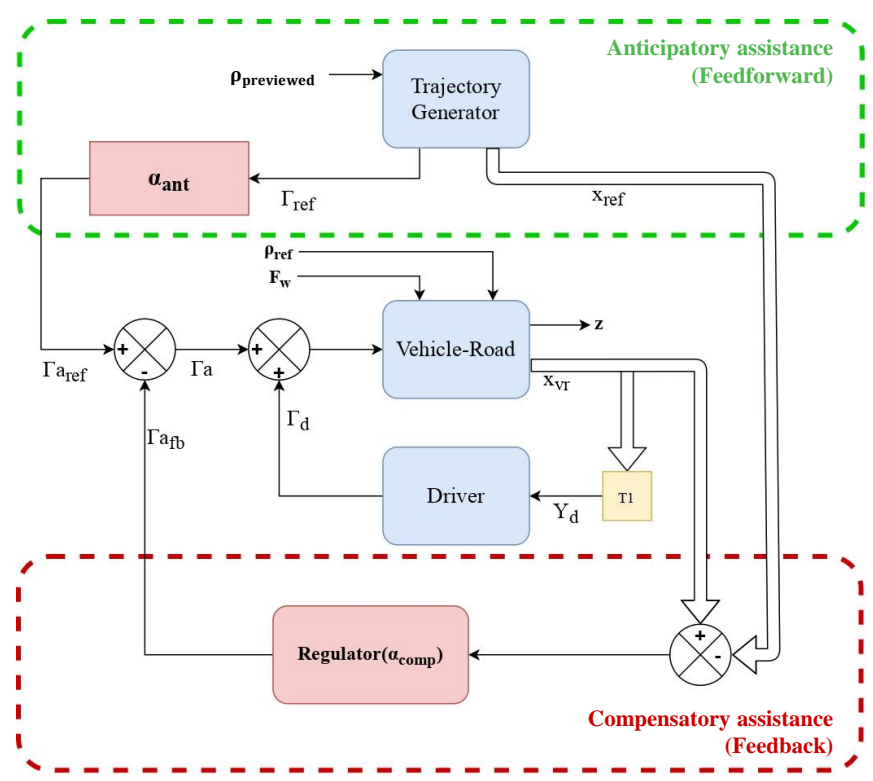

Fig. 4. Design strategy of the haptic guidance system.

the vehicle's position in the lane. It consisted of an $H_{2} / H_{\infty}$ static output feedback that controlled the steering against disturbances and uncertainties:

$$
\Gamma_{f b}=K_{f b}\left(x_{v r}-x_{r e f}\right)
$$

Similarly, the applied compensatory torque control was determined according to the sharing level, $\alpha_{\text {comp }}$, of the compensatory part: $\Gamma_{a_{f b}}=\Gamma_{f b}\left(\alpha_{c o m p}\right)$.

In this study, both sharing levels were fixed at $50 \%$. This configuration results in a system that delivers clear haptic guidance, although without any action from the human driver it will eventually leave the lane during some curves.

\section{Participants}

Five participants took part in the experiment. They were recruited from students and staff of the Laboratory of Digital Sciences in Nantes and Institute Mines-Télécom Atlantique Nantes. All participants possessed a valid driver's license with at least three years of driving experience. The participants had no known medical issues that could affect their driving skills. None of them had ever experienced a haptic guidance system.

\section{Scenarios}

The track used in the experiment is shown in Fig. 3 (right). It was a two-lane road with a lane width of $3.5 \mathrm{~m}$. All curves were Euler spirals with continuous changes in the curvature of the road. At the beginning of the experiment, all participants were provided with a brief introduction to the simulator, including the haptic guidance system. They were then instructed to drive in two different scenarios in random order: with and without the haptic guidance system. During the task, the vehicle longitudinal speed was fixed at $64 \mathrm{~km} / \mathrm{h}(18 \mathrm{~m} / \mathrm{s})$ so that they only needed to control the steering wheel. They were asked to drive in the right-hand

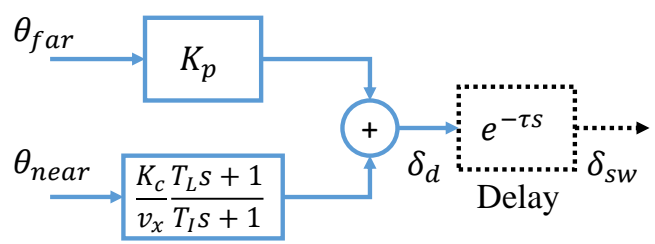

Fig. 5. Visual model with delay for identification.

lane without making any lane changes for $10 \mathrm{~min}$ in each scenario, which equaled to almost two laps on the track.

\section{Model Parameter Identification}

It is difficult to identify the entire model directly, for the following reasons: 1) the target steering-wheel angle is not measurable; 2) the internal model is implicitly affected by the haptic feedback; 3) some parameters are weakly identifiable with the entire model. Thus, the model is separated into three parts that are identified one by one, sequentially. The data collected from the experiment are also equally divided into two parts, one for identification and another for validation.

\section{A. Visual Model Identification}

The visual model considered, shown in Fig. 5, borrows from that in Fig. 1. The main problem is that the target steering-wheel angle $\delta_{d}$ that the driver is supposed to derive from the visual angle is unknown. The only relevant signal measured was the actual angle of the steering wheel, $\delta_{S W}$. Assuming that there is only a delay between the two angles, it is possible to obtain the visual model with delay by identification using $\delta_{S W}$ as an output, which yields the desired parameters $\left(K_{p}, K_{c}, T_{I}, T_{L}\right)$.

One minimal realization of the visual model with delay (approximated by a first-order system) is written as follows:

$$
\begin{aligned}
& \dot{x}=A x+B u \\
& y=C x+D u
\end{aligned}
$$

with

$$
\begin{aligned}
y & =\delta_{d}, u=\left[\begin{array}{ll}
\theta_{\text {far }} & \theta_{\text {near }}
\end{array}\right]^{T}, \\
A & =\left[\begin{array}{cc}
-\frac{1}{T_{I}} & 0 \\
-\frac{1}{\tau} \frac{K_{c}}{v_{x}}\left(\frac{T_{L}}{T_{I}}-1\right) & -\frac{1}{\tau}
\end{array}\right] B=\left[\begin{array}{cc}
0 & \frac{1}{T_{I}} \\
\frac{1}{\tau} K_{p} & \frac{1}{\tau} \frac{K_{c}}{v_{x}} \frac{T_{L}}{T_{I}}
\end{array}\right] \\
C & =\left[\begin{array}{lll}
0 & 1
\end{array}\right] \\
D & =\left[\begin{array}{ll}
0 & 0
\end{array}\right]
\end{aligned}
$$

The input signals highlighted in Fig. 6 were approximated as follows, with $D_{f a r}=18 \mathrm{~m}$ and $l_{s}=5 \mathrm{~m}$.

$$
\theta_{\text {far }} \approx D_{\text {far }} \times \rho, \theta_{\text {near }} \approx-y_{L} / l_{s}
$$

The identification was performed using the prediction error minimization (PEM) [20] method with data from the experiment. The system identification toolbox in MATLAB was used to compute the identification results. The criterion was:

$$
J=\sum_{k=1}^{N} e_{v}^{2}[k]
$$




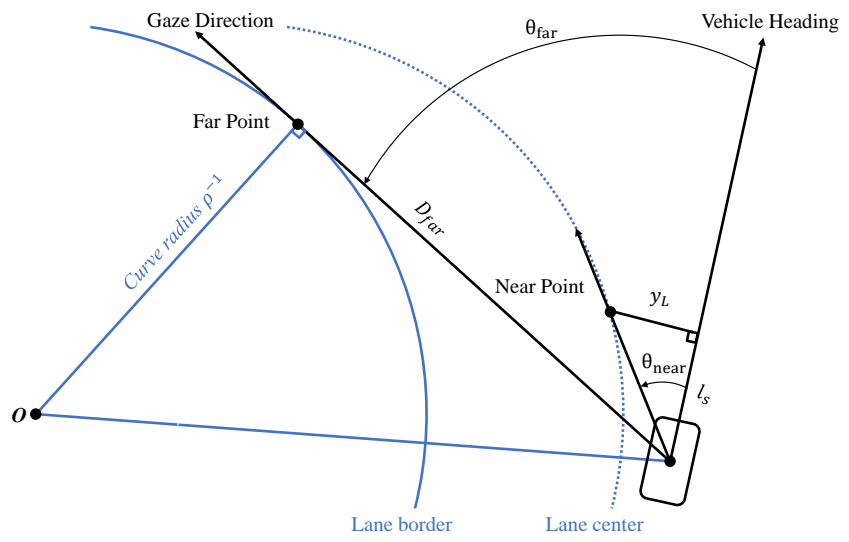

Fig. 6. Approximation of the far point angle and the near point angle.

where $e_{v}[k]$ represents the difference between the measured output and predicted output of the model $\hat{\delta}_{S W}$ :

$$
e_{v}[k]=\delta_{S W}[k]-\hat{\delta}_{S W}[k]
$$

A potential problem with using the actual steering-wheel angle to identify the visual model is that when a haptic guidance system is used for driving, the actual steering-wheel angle represents the joint effort of the human driver and the haptic guidance system. The identified visual model would in this case be a projection of the dynamics of the driverautomation system. To avoid this problem, it is necessary that the visual model of the human driver is invariant. For this purpose and for each participant, the visual model was identified from the data of the scenario without haptic guidance, and kept as-is in the scenario with haptic guidance to identify the rest of the driver model.

\section{B. Driver Internal Model Identification}

As the vehicle's longitudinal speed was fixed at $18 \mathrm{~m} / \mathrm{s}$ in the experiment, only the static gain $K_{I}$ needed to be identified in the driver internal model. This could be achieved by fixing the value of parameters in the visual model and minimizing the following criterion:

$$
J=\sum_{k=1}^{N} e_{I}^{2}[k]
$$

where

$$
e_{I}[k]=\Gamma_{d}[k]-\hat{\Gamma}_{I}[k]
$$

\section{Explicit Haptic Feedback Loop Identification}

Once the visual model and the driver internal model had been identified, the resulting parameter values could be used to identify the entire model by focusing on the explicit haptic feedback loop. One minimal realization of the entire model can be written as follows:

$$
\begin{aligned}
& \dot{x}=A x+B u \\
& y=C x+D u
\end{aligned}
$$

TABLE II

IDENTIFIED VISUAL MODEL

\begin{tabular}{c|c|c|c|c|c|c}
\hline \hline Participant & $K_{p}$ & $K_{c}$ & $T_{I}$ & $T_{L}$ & $\tau$ & $F I T(\%)$ \\
\hline P1 & 3.69 & 2.63 & 0.38 & 7.19 & 0.57 & 85.61 \\
P2 & 3.82 & 3.69 & 0.45 & 5.80 & 0.56 & 79.45 \\
P3 & 3.63 & 2.49 & 0.44 & 7.00 & 0.53 & 84.86 \\
P4 & 3.67 & 2.15 & 0.70 & 6.47 & 0.61 & 82.70 \\
P5 & 3.79 & 2.99 & 0.62 & 8.16 & 0.62 & 86.79 \\
\hline \hline
\end{tabular}

with

$$
\begin{aligned}
& y=\Gamma_{d}, u=\left[\begin{array}{ccc}
\theta_{f a r} & \theta_{\text {near }} & \Gamma_{f b}
\end{array}\right]^{T}, \\
& -\frac{1}{T_{I}} \\
& \left.\begin{array}{ccc}
0 & 0 & 0 \\
-K_{I} v_{x} \frac{1}{T_{N}} \frac{K_{c}}{v_{x}}\left(\frac{T_{L}}{T_{I}}-1\right) & -\frac{1}{T_{N}} & 0 \\
0 & -\frac{K_{f b}}{T_{f b}} & -\frac{1}{T_{f b}}
\end{array}\right] \\
& B=\left[\begin{array}{ccc}
0 & \frac{1}{T_{I}} & 0 \\
K_{I} v_{x} \frac{1}{T_{N}} K_{p} & K_{I} v_{x} \frac{1}{T_{N}} \frac{K_{c}}{v_{x}} \frac{T_{L}}{T_{I}} & 0 \\
0 & 0 & \frac{K_{f b}}{T_{f b}}
\end{array}\right] \\
& C=\left[\begin{array}{ccc}
0 & 1 & 1
\end{array}\right] D=\left[\begin{array}{lll}
0 & 0 & 0
\end{array}\right]
\end{aligned}
$$

Similar to the identification of the visual model, the results were also calculated using the PEM method implemented in the system identification toolbox of MATLAB. The only two parameters to be identified at this stage were $K_{f b}$ and $T_{f b}$. The other parameters were fixed at their nominal values, which were obtained at previous identification stages. The criterion was similar to (10), except the error was the difference between the measured driver steering torque and the predicted steering torque of the model:

$$
J=\sum_{k=1}^{N} e_{d}^{2}[k], e_{d}[k]=\Gamma_{d}[k]-\hat{\Gamma}_{d}[k]
$$

\section{RESULTS}

\section{A. Visual Model with Delay}

As detailed in section IV-A, the visual model was identified first, based on the data recorded ad hoc when the participants were driving alone. The identified parameters of the model, including the time delay, $\tau$, are listed in Table II. The FIT value, which indicates the difference between the predicted model output and the measured output, is calculated as follows:

$$
F I T=\left(1-\frac{\left\|\delta_{S W}-\hat{\delta}_{S W}\right\|_{2}}{\left\|\delta_{S W}-m_{\delta_{S W}}\right\|_{2}}\right) \times 100 \%
$$

where $m_{\delta_{S W}}$ is the arithmetic mean of $\delta_{S W}$. The identification results converged to almost the same ranges in value for all participants, with the model explaining on average $83.88 \%$ of the actual steering-wheel angle. No parameter showed an abnormal value. The variation of model parameters, especially for the near-point angle input, could imply different driving styles. In addition, the values of delay were 
TABLE III

IDENTIFIED DRIVER INTERNAL MODEL

\begin{tabular}{c|c|c||c|c}
\hline \hline \multirow{2}{*}{ Participant } & \multicolumn{2}{|c||}{ Without Haptic Guidance } & \multicolumn{2}{c}{ With Haptic Guidance } \\
\cline { 2 - 5 } & $K_{I}$ & $F I T(\%)$ & $K_{I}$ & $F I T(\%)$ \\
\hline P1 & 0.214 & 83.46 & 0.128 & 51.09 \\
P2 & 0.211 & 81.48 & 0.157 & 64.33 \\
P3 & 0.217 & 85.42 & 0.129 & 59.51 \\
P4 & 0.213 & 82.20 & 0.128 & 64.80 \\
P5 & 0.210 & 82.42 & 0.156 & 51.18 \\
\hline \hline
\end{tabular}

close because they mainly represented the transport delay of the simulator (computation, graphics, sensors etc.) [8].

\section{B. Driver Internal Model}

The identified values of the driver internal gain, $K_{I}$, based on the data without and with the haptic guidance system, are listed in Table III. These values enable a comparison of the torque output predicted by the identified driver internal model, $\hat{\Gamma}_{I}$, and the measured driver steering torque, $\Gamma_{d}$, which is the FIT value shown in the table, calculated as follows:

$$
F I T=\left(1-\frac{\left\|\Gamma_{d}-\hat{\Gamma}_{I}\right\|_{2}}{\left\|\Gamma_{d}-m_{\Gamma_{d}}\right\|_{2}}\right) \times 100 \%
$$

where $m_{\Gamma_{d}}$ is the arithmetic mean of $\Gamma_{d}$.

A significant decrease in the gain of the driver internal model was observed when the participants were driving with the haptic guidance. This was reasonable, as one of the main objectives of the haptic guidance system is to reduce the driver's effort in controlling the vehicle by providing additional torque. It also demonstrates that, as hypothesized, the participants adjusted their internal model to cooperate with the haptic guidance during the experiment. In fact, all five participants successfully completed the experiment without having any difficulty in maintaining the vehicle in the lane. For all participants, the reduction of the gain $K_{I}$ was around $60 \%$ to $70 \%$, which is related to the $50 \%$ sharing level chosen for the haptic guidance system in the experiment. The difference may suggest that participants did not fully rely on the system.

There was also a significant difference in the FIT values between the two conditions. Without the haptic guidance, the predicted torque output from the driver internal model was rather close to the measured driver steering torque. This was not the case with the haptic guidance. To investigate this difference, a residual analysis was performed. As an example, Fig. 7 shows the residual of the driver internal model (i.e., $\Gamma_{d}-\hat{\Gamma}_{I}$ ) of participant P1 in both conditions. Without haptic guidance, the residual was almost white noise-because in this situation the haptic feedback was merely self-aligning torque. The self-aligning torque is usually modelled as being approximately proportional to the steering-wheel angle [21]. Thus, a driver internal model in the form of a simple gain is almost accurate. However, this is not the case when the haptic guidance is part of the haptic feedback. In that case, the relationship between the haptic feedback and the steering
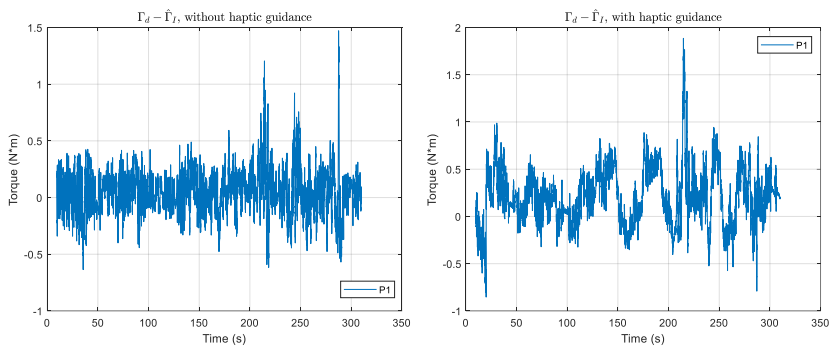

Fig. 7. Residual of the driver internal model for P1. Left: without haptic guidance; right: with haptic guidance.

TABLE IV

Identified Explicit Haptic FeEdback Model

\begin{tabular}{c|c|c|c||c|c|c}
\hline \hline \multirow{2}{*}{ Participant } & \multicolumn{3}{|c||}{ Without Haptic Guidance } & \multicolumn{3}{c}{ With Haptic Guidance } \\
\cline { 2 - 7 } & $K_{f b}$ & $T_{f b}$ & $F I T(\%)$ & $K_{f b}$ & $T_{f b}$ & $F I T(\%)$ \\
\hline P1 & 0.92 & 0.014 & 91.11 & 0.97 & 0.023 & 86.34 \\
P2 & 0.90 & 0.012 & 91.83 & 0.90 & 0.028 & 88.97 \\
P3 & 0.86 & 0.013 & 91.66 & 0.96 & 0.022 & 86.26 \\
P4 & 0.92 & 0.014 & 91.79 & 0.91 & 0.027 & 85.38 \\
P5 & 0.89 & 0.016 & 91.36 & 0.97 & 0.027 & 88.34 \\
\hline \hline
\end{tabular}

wheel angle depends on the design strategy of the haptic guidance system and is difficult to predict only with the driver internal model. This leads to the difference between FIT values and the useful information left in the residual, which is explained by the explicit haptic feedback loop.

\section{Explicit Haptic Feedback Loop}

The identified explicit haptic feedback loop, without and with the haptic guidance system, is listed in Table IV. The FIT is calculated similarly as (15), where the predicted model output is $\hat{\Gamma}_{d}$ and the measured output is $\Gamma_{d}$.

The FIT values of the model with the explicit haptic feedback loop showed a close match between the predicted and measured driver steering torque, under both conditions (either with or without the haptic guidance). Compared with the results in Table III, a distinct improvement is evident, especially when haptic guidance torque was part of the haptic feedback. These results indicate that this model structure was valid in both situations, although with haptic guidance there was still a substantial loss of around $3 \%$ to 5\%. This point could be investigated in future research.

A similar gain between the two experimental conditions was obtained for the explicit haptic feedback loop. The time constant was almost doubled in the case of haptic guidance but remained smaller than the neuromuscular time constant fixed, $T_{N}$, which was $0.23 \mathrm{~s}$. The difference between $T_{f b}$ and $T_{N}$ could correspond to the fact that control by spinal feedback pathways (30-40 ms for the arm muscles) is more efficient and faster than control by supraspinal pathways (i.e. the visual system) [22]. It should be noted, however, that the $T_{f b}$ value could also be artificially increased or decreased by potential delays in the dynamics of the torque sensors. The difference in $T_{f b}$ between the two conditions is probably related to the predictive property of the haptic guidance 

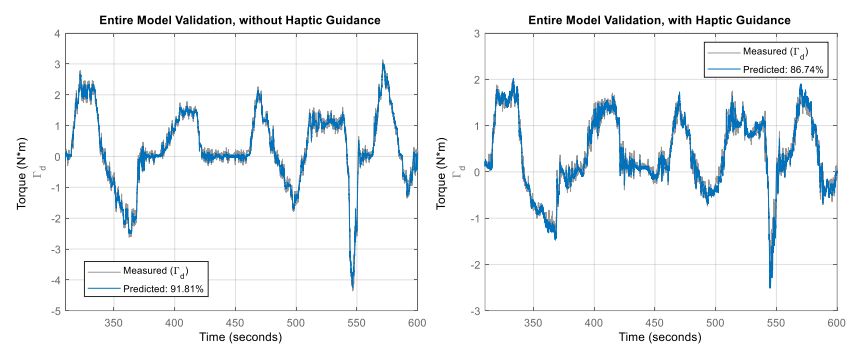

Fig. 8. Validation of the entire driver model for P1. Left: without haptic guidance; right: with haptic guidance.

system. As part of its design strategy, the system anticipates changes in road curvature and can generate guidance torque even before the driver does. The haptic feedback that includes the assistance torque therefore occurs before the haptic feedback includes only the self-aligning torque, allowing the human driver more time to react.

Once each part of the model was identified, validation of the entire model using experimental data was performed to verify the model prediction. As an example, Fig. 8 shows the results of the validation of participant P1 under both conditions. The figure shows that the driver model output predicts well the driver steering torque measured in the validation data, whether driving without or with the haptic guidance system.

\section{CONCLUSION}

In this article, a new cybernetic model of the driver in the steering task is proposed, with a reconsidered neuromuscular sub-model. The objective is to model the driver's steering control behavior, especially when a haptic guidance system is active. The haptic feedback signal, which includes both guidance and self-aligning torque, is used as a key input to the new model. It intervenes in the steering control, both by adapting the gain of the internal model to the compliance of the steering system (implicit feedback) and by explicit feedback after comparison with the target torque derived from the visual system. The results from our identification study show a good fit between the predicted and measured steering torque by the driver. Future work will focus on the model with different haptic steering systems set at different split levels. Additional tests involving more drivers will be conducted to examine how the proposed model can help to further understand how drivers interact with haptic guidance systems.

\section{ACKNOWLEDGMENT}

This work was supported by RFI Atlanstic 2020, funded by Région Pays de la Loire.

\section{REFERENCES}

[1] M. Steele and R. B. Gillespie, "Shared Control between Human and Machine: Using a Haptic Steering Wheel to Aid in Land Vehicle Guidance," Proceedings of the Human Factors and Ergonomics Society Annual Meeting, vol. 45, no. 23, pp. 1671-1675, 2001.

[2] D. A. Abbink and M. Mulder, "Exploring the dimensions of haptic feedback support in manual control," Journal of Computing and Information Science in Engineering, vol. 9, no. 1, pp. 1-9, 2009.

[3] P. G. Griffiths and R. B. Gillespie, "Sharing control between humans and automation using haptic interface: Primary and secondary task performance benefits," Human Factors, vol. 47, no. 3, pp. 574-590, 2005, pMID: 16435698.

[4] M. Mulder, D. A. Abbink, and E. R. Boer, "Sharing control with haptics: Seamless driver support from manual to automatic control," Human Factors, vol. 54, no. 5, pp. 786-798, 2012.

[5] Z. Wang, R. Zheng, T. Kaizuka, K. Shimono, and K. Nakano, "The effect of a haptic guidance steering system on fatigue-related driver behavior," IEEE Transactions on Human-Machine Systems, vol. 47, no. 5, pp. 741-748, 2017.

[6] L. Saleh, P. Chevrel, F. Claveau, J. F. Lafay, and F. Mars, "Shared steering control between a driver and an automation: Stability in the presence of driver behavior uncertainty," IEEE Transactions on Intelligent Transportation Systems, vol. 14, no. 2, pp. 974-983, 2013.

[7] B. Pano, P. Chevrel, and F. Claveau, "Anticipatory and compensatory e-assistance for haptic shared control of the steering wheel," in 2019 18th European Control Conference (ECC), 2019, pp. 724-731.

[8] F. Mars, L. Saleh, P. Chevrel, F. Claveau, and J.-F. Lafay, "Modeling the visual and motor control of steering with an eye to shared-control automation," Proceedings of the Human Factors and Ergonomics Society Annual Meeting, vol. 55, no. 1, pp. 1422-1426, 2011.

[9] L. Saleh, P. Chevrel, F. Mars, J. F. Lafay, and F. Claveau, "Human-like cybernetic driver model for lane keeping," in Proceedings of the 18th IFAC World Congress, 2011, pp. 4368-4373.

[10] F. Mars and P. Chevrel, "Modelling human control of steering for the design of advanced driver assistance systems," Annual Reviews in Control, vol. 44, no. Supplement C, pp. 292-302, 2017.

[11] L. Saleh, "Contrôle Latéral Partagé d'un Véhicule Automobile," Ph.D. dissertation, Ecole Centrale de Nantes (ECN), apr 2012.

[12] P. Hermannstädter and B. Yang, "Driver Distraction Assessment Using Driver Modeling," in 2013 IEEE International Conference on Systems, Man, and Cybernetics, oct 2013, pp. 3693-3698.

[13] A. Ameyoe, F. Mars, P. Chevrel, E. Le Carpentier, and H. Illy, "Estimation of driver distraction using the prediction error of a cybernetic driver model," Proceedings of the driving simulation conference Europe 2015, pp. 13-18, 2015.

[14] E. Donges, "A Two-Level Model of Driver Steering Behavior," Human Factors: The Journal of the Human Factors and Ergonomics Society, vol. 20, no. 6, pp. 691-707, dec 1978 .

[15] D. D. Salvucci and R. Gray, "A two-point visual control model of steering," Perception, vol. 33, no. 10, pp. 1233-1248, 2004.

[16] D. Toffin, G. Reymond, A. Kemeny, and J. Droulez, "Role of steering wheel feedback on driver performance: driving simulator and modeling analysis," Vehicle System Dynamics, vol. 45, no. 4, pp. 375-388, 2007.

[17] E. R. Kandel, J. H. J. H. Schwartz, T. M. Jessell, S. Siegelbaum, A. J. Hudspeth, and S. Mack, Principles of neural science, 5th ed. McGraw-Hill Education / Medical, 2013.

[18] A. Pick, "Neuromuscular dynamics and the vehicle steering task," Ph.D. dissertation, University of Cambridge, 2004.

[19] D. A. Abbink, M. Mulder, and M. M. Van Paassen, "Measurements of muscle use during steering wheel manipulation," in Conference Proceedings - IEEE International Conference on Systems, Man and Cybernetics, 2011, pp. 1652-1657.

[20] L. Ljung, System Identification: Theory for the User, 2nd ed., ser. Prentice Hall information and system sciences series. Prentice Hall PTR, 1999.

[21] N. Minoiu Enache, "Assistance préventive à la sortie de voie," Theses, Université d'Evry-Val d'Essonne, Nov. 2008. [Online]. Available: https://tel.archives-ouvertes.fr/tel-00364073

[22] E. De Vlugt, "Identification of Spinal Reflexes," Ph.D. dissertation, Delft University of Technology, 2004. 